\section{Beatmungsentwöhnung in der neurologisch-neurochirurgischen Frührehabilitation}

\author{
M. Pohl
}

\section{Zusammenfassung}

Beatmete Patienten werden der neurologisch-neurochirurgischen Frührehabilitation (NNFR) in aller Regel im Rahmen eines prolongierten Weanings zugewiesen. Die Ursachen der Langzeitbeatmung bei diesen Patienten sind nicht nur neurologisch, sondern häufig multifaktoriell. Hierbei kommt der im Vordergrund stehenden oder zusätzlich begleitenden Critical-Illness-Polyneuropathie und -myopathie große Bedeutung zu. Neurologische Patienten im prolongierten Weaning benötigen spezielle Behandlungskonzepte (motorische Rehabilitation, Trachealkanülen- und Dysphagiemanagement etc.), die sich in den Strukturen NNFR optimal abbilden lassen.

Bei der Entwöhnung langzeitbeatmeter Patienten wird unabhängig von der Ursache des prolongierten Weanings der Einsatz von Weaningprotokollen empfohlen. Andere spezielle Weaningmethoden (insbesondere am Beatmungsgerät) werden kontrovers diskutiert.

Die Weaningergebnisse der neurologischen Weaningzentren der NNFR sind mit 60-80\% erfolgreichem Weaning, 5-20\% Weaningversagen mit der Notwendigkeit zur außerklinischen Beatmung und 6-23\% Sterblichkeit vergleichbar mit den Ergebnissen nationaler Studien aus pneumologischen Weaningzentren und internationalen Studien multidisziplinärer Weaningzentren. Diese Vergleichbarkeit ist aber aufgrund extrem unterschiedlicher medizinischer, organisatorischer, wirtschaftlicher und sozialrechtlicher Voraussetzungen der unterschiedlichen Weaningstrukturen stark eingeschränkt.

Das Weaning in spezialisierten neurologischen Abteilungen oder Kliniken der NNFR stellt nach Auffassung des Autors insgesamt einen wesentlichen Baustein im Gesamtbehandlungskonzept von Patienten mit primären und sekundären neurologisch-neurochirurgischen Erkrankungen dar.

Schlüsselwörter: Beatmung, Langzeitbeatmung, prolongiertes Weaning, neurologische Weaningzentren
Neurol Rehabil 2016; 22(3): 187-191

(C) Hippocampus Verlag 2016

DOI 10.14624/NR150816.002
HELIOS Klinik Schloss Pulsnitz $\mathrm{GmbH}$, Fachklinik für NeurologischNeurochirurgische Rehabilitation

\section{Einleitung}

In der neurologisch-neurochirurgischen Frührehabilitation (NNFR) erfolgt seit Jahrzehnten die Versorgung sehr schwer erkrankter neurologischer Patienten [33]. Durch die Optimierung von Notfall- und Intensivversorgung überleben heute viel mehr Patienten nach SchädelHirn-Trauma, Reanimationen oder Multiorganversagen nach Sepsis, die dann schwere neurologische Ausfälle davontragen [32]. Auch die Fortschritte in den klinischen Fächern Neurologie und Neurochirurgie sichern vielen Patienten das Überleben. Diese Patienten sind oft so schwer betroffen, dass sie während des Aufenthaltes im Akutkrankenhaus nicht erfolgreich von der Beatmung entwöhnt werden können. Beatmete Patienten, die zum Weaning im Rahmen einer NNFR verlegt wurden, waren im Mittel schon mehr als drei Wochen ( $36 \pm 31$ Tage) im primär versorgenden Krankenhaus beatmet [19]. Eine intensivmedizinische Behandlung dieser Dauer führt bereits bei nicht neurologischen Patienten zu einer mus- kulären Schwäche, die durch Inaktivität sowie CriticalIllness-Neuropathie bzw. -Myopathie bedingt ist [18]. Neurologisch-neurochirurgische Frührehabilitanden weisen zudem noch durch zentral bedingte Lähmungen oder neuromuskuläre Erkrankungen eine erhebliche Immobilität und Abhängigkeit von pflegerischer Hilfe auf, die den Weaningprozess zusätzlich erheblich erschweren [20].

Dass die Entwöhnung von der maschinellen Beatmung auch in Zentren der NNFR erfolgreich verläuft, belegen aktuelle, nicht kontrollierte Studien [9, 19, 21, 25]. Hinsichtlich der technischen Abläufe sowie der Pathophysiologie des Weaningversagens sei in diesem Zusammenhang auf die pneumologische S2k-Leitlinie zum prolongierten Weaning verwiesen [31], hier ergeben sich beim Weaning in der NNFR keine wesentlichen Abweichungen. Die neurologisch-neurochirurgischen Patienten benötigen aber ein besonderes Setting mit ergänzenden Strukturen, das nur in einem Zentrum der NNFR bereitgestellt werden kann. Durch die Möglich- 
Weaning from prolonged mechanical ventilation in early neurological and neurosurgical rehabilitation (ENNR)

M. Pohl

\section{Abstract}

The majority of mechanically ventilated patients are referred for early neurological and neurosurgical rehabilitation (ENNR) during the course of prolonged weaning. The causes for long-term mechanical ventilation are not only neurological, but frequently multifactorial as well. In this context, critical illness polyneuropathy and myopathy often lead to prolonged weaning or complicate the weaning process.

Neurological patients with prolonged weaning need special treatment concepts (e.g., motor rehabilitation, need for a tracheal cannula, or management of dysphagia) that are relevant to ENNR.

The use of weaning protocols is always recommended in prolonged weaning irrespective of the indication for long-term mechanical ventilation. Other special weaning methods (especially the use of mechanical ventilators) are controversially discussed.

Weaning outcomes in ENNR are: 60-80\% successful weaning; 5-20\% weaning failure; and 6-23\% mortality during ENNR. These results are comparable to the outcome of German pneumological weaning centers and international multidisciplinary weaning centers. However, the comparability is limited by heterogeneous medical, organizational, economic, and social conditions.

In the opinion of the author, weaning from prolonged mechanical ventilation at ENNR centers is an essential part of the overall treatment concept for patients with primary and secondary neurological and neurosurgical diseases.

Keywords: mechanical ventilation, prolonged mechanical ventilation, prolonged weaning, neurological weaning units

Neurol Rehabil 2016; 22(3): 187-191

(C) Hippocampus Verlag 2016 im Verlauf dann eher multifaktoriell. Oft findet sich zusätzlich eine Atemmuskelschwäche im Rahmen einer allgemeinen Muskelschwäche mit begleitender CriticalIllness-Polyneuropathie (CIP) bzw. -myopathie (CIM). Sie wird bei $25 \%$ der Patienten mit einer Beatmungsdauer von mehr als sieben Tagen sowie bei $71 \%$ der Patienten nach schwerer Sepsis beobachtet und ist signifikant mit einer verlängerten Beatmungs- sowie Hospitalisierungsdauer assoziiert [16, 18, 24]. Bei Patienten mit einer Beatmungsdauer von mehr als 14 Tagen und fehlender anderer neurologischer Diagnose lässt sich in mehr als 90\% der Fälle eine klinisch wahrscheinliche bzw. mögliche CIP/CIM als ursächlich für die anhaltende Beatmungspflicht finden [18].

Neben den sekundären neurologischen Folgen der Langzeitintensivtherapie sind primär neurologische Krankheitsbilder häufige Ursache für ein prolongiertes Weaning $[4,16,19,25]$. So tritt bei $27 \%$ der beatmeten Patienten mit neurologisch-neurochirurgischen Grunderkrankungen ein »Weaningversagen« auf [7, 19]. Auch andere neurologische und psychische Folgen der Intensivbehandlung (z. B. Delir, Posttraumatische Belastungsstörung) haben einen ungünstigen Einfluss auf das Weaning der Patienten [16].

Es besteht allgemeiner Konsens darüber, dass Patienten mit Langzeitbeatmung in dafür spezialisierte Einrichtungen (Weaningzentren) verlegt werden sollten [5, 14, 19, 31]. Dies geschieht zunehmend früher nach Primärtherapie [17]. Die Patienten sind bei Übernahme in ein Weaningzentrum meist in schlechtem funktionellen Zustand [19, 27, 31].

Neurologische Patienten mit prolongiertem Weaning, die nicht in der Primärversorgung von der Beatmung entwöhnt werden konnten, benötigen eine nahtlose Fortführung rehabilitativer Maßnahmen in der Sekundärversorgung unter der Berücksichtigung einer vorhandenen intensivmedizinischen Betreuung in einem spezialisierten Zentrum. Diese Zentren der NNFR erfüllen bei der Versorgung langzeitbeatmeter Patienten spezielle Aufgaben und Anforderungen. Einige wesentliche Anforderungen seien wie folgt hervorgehoben:

- Möglichkeiten zur (erneuten) intensivmedizinischen Behandlung bei Komplikationen (z.B. Pneumonie mit erneuter Beatmungspflicht)

- Umgang mit absaugpflichtigem Tracheostoma

- Dysphagiemanagement

- Konzepte zur Behandlung von Patienten mit hirnorganischem Psychosyndrom und speziellen neurokognitiven Funktionsstörungen neurologischen Krankheiten (z.B. hohes Querschnittsyndrom, schwere Polyneuropathien, Hirnstammsyndrome oder Großhirnerkrankungen) die Folgen eines »acute respiratory distress syndrome« (ARDS), postoperative Zustände mit Delir [14], Exazerbationen einer »chronic obstructive pulmonary disease (COPD) bzw. einer chronischen Lungenerkrankung sein $[1,6,8,13$, 31]. Die Ursache der anhaltenden Beatmungspflicht ist
- Spezialisierung zur Behandlung von Patienten mit Querschnittsyndromen

- Behandlungskonzepte zur Wund- und Dekubitustherapie

- Konzepte und Therapieansätze zur systematischen Steigerung der Selbstständigkeit in den Aktivitäten des täglichen Lebens und der Teilhabe 
- Alle gängigen Konzepte der modernen Neurologischen (Früh-)Rehabilitation mit Überleitungsmanagement in die weiterführende Rehabilitation (Phasen C-D) und die außerklinische Versorgung

- Konzept zur Betreuung von dauerhaft beatmeten Patienten mit sogenannten »Heimbeatmungsgeräten« mit Management zur Vorbereitung auf die außerklinische Beatmung

\section{Studien zum Weaning in der neurologischen Frührehabilitation}

Bisher gibt es keine kontrollierten Studien zum Weaning neurologischer Patienten in der NNFR. Die veröffentlichten Studien und Beiträge beschreiben das Vorgehen beim Weaning oder sind Expertenmeinungen und Konzeptdarstellungen [4, 16], retrospektive Datenerhebungen [19, 21, 25], spezielle Konzepte beim Weaning [17] oder Darstellungen von Weaningverläufen in der NNFR [3, 9].

Die größte Studie in diesem Kontext ist eine retrospektive Untersuchung der Arbeitsgemeinschaft (AG) NNFR, für die die Daten von 1.486 zum Weaning aufgenommenen Patienten aus sieben untersuchten Kliniken der NNFR im Jahr 2009 ausgewertet wurden [19]. Die Patientencharakteristika und die wichtigsten Ergebnisse dieser Studie der AG NNFR [19] sind Tabelle $1 \mathrm{zu}$ entnehmen. Die Tabelle 2 zeigt die Ergebnisse in verschiedenen Weaningzentren der NNFR aus der aktuellen Literatur im Vergleich. Die Tabelle 2 zeigt, dass die Weaningergebnisse der Studien aus dem Gebiet der NNFR vergleichbar sind.

Ponfick und Mitarbeiter zeigten zudem in einer retrospektiven Analyse eines neurologischen Weaningzentrums, dass sich die Weaningdauer negativ auf das funktionelle Outcome von Patienten auswirkt. Dies konnte in dieser Studie aber durch einen längeren stationären Reha-Aufenthalt im Anschluss an das erfolgreiche Weaning erfolgreich kompensiert werden [23].

Der internationale Vergleich der Entwöhnungsraten der unterschiedlichen Weaningzentren (neurologisch, pneumologisch und gemischt) ist insbesondere aufgrund der differierenden Aufnahmekriterien und der uneinheitlichen Definition bezüglich eines erfolgreichen Weanings schwierig. Die geforderte durchgehende Spontanatmung für ein erfolgreiches Weaning liegt zwischen 24 Stunden [28] und 7 Tagen [1,6,26], andere Autoren geben keine Definition an $[10,13,25]$. Zum Teil wird auch eine sich an die Entwöhnung anschließende nicht invasive Beatmung als erfolgreiches Weaning angesehen [2, 28-30]. Zudem ist eine vergleichende Beurteilung der Mortalität unterschiedlicher Weaningzentren nur unter Berücksichtigung des Vorgehens bei Therapiebegrenzung möglich, das meist nicht transparent ist. Der internationale Vergleich der Ergebnisse ist weiterhin nur eingeschränkt möglich, da die Behandlungsmöglichkeiten bei Komplikationen und die Angaben zur Letalität aus medizinischen, organisatorischen, wirtschaftlichen und sozialrechtlichen Gründen sehr heterogen sind [17].

\section{Entwöhnungsstrategien und Beatmungsprotokolle}

Die Entwöhnung von der maschinellen Beatmung nimmt etwa 40-50\% der Gesamtbeatmungsdauer in Anspruch. Mit Dauer der maschinellen Beatmung steigen Mortalität und Komplikationsrate [5, 31]. Die sichere und zügige Entwöhnung vom Respirator hat daher oberste Priorität. Zur Frage, welche Methoden die sichere und zügige Entwöhnung vom Beatmungsgerät gewährleisten und ob bestimmte Vorgehensweisen oder Beatmungsmodi das Weaning beschleunigen, gibt es keine ausreichenden Daten im Kontext des Weanings in der NNFR. Auch bei nicht neurologischen Patienten bzw. nicht selektierten Patientengruppen wird diese Frage kontrovers diskutiert (s. auch [31]). Ziel der Entwöhnung von der Beatmung bei neurologischen Patienten ist die selbstständige Atmung ohne Geräteunterstützung. Die nicht invasive Beatmung wird als Deeskalationstherapie der Beatmung bei guter Schluckfunktion und weiterhin bestehender Atempumpeninsuffizienz genutzt [4].

Bei der Entwöhnung langzeitbeatmeter Patienten wird der Einsatz von Weaningprotokollen allgemein empfohlen $[15,31]$. Diese sollten eine stufenweise Verlängerung der Spontanatmungsversuche beinhalten [14,

Tab. 1: Patientencharakteristika und wichtige Ergebnisse der Studie der AG NNFR [19]

\begin{tabular}{|c|c|}
\hline Alter & $63,7 \pm 14,8$ Jahren (Range 18-96 Jahre) \\
\hline $\begin{array}{l}\text { mittlere } \\
\text { Beatmungsdauer } \\
\text { vor Übernahme } \\
\text { in die jeweiligen } \\
\text { Weaningzentren }\end{array}$ & $35,8 \pm 30,6$ Tagen (Range $1-256$ Tage) \\
\hline $\begin{array}{l}\text { Ursachen für die } \\
\text { Langzeitbeatmung }\end{array}$ & $\begin{array}{l}\text { - } \text { neurologisch } 69,2 \% \text { (davon } 52,6 \% \text { zentralnervös, } \\
45,2 \% \text { peripher-neurologisch, } 2,2 \% \text { gemischt) } \\
\text { - pulmonal } 22,8 \% \\
\text { - } \text { kardial } 3,0 \% \\
\text { - unklar } 5,0 \%\end{array}$ \\
\hline Diagnosen & $\begin{array}{l}\text { - Hirninfarkt } 17,2 \% \\
\text { - } \text { ICB oder SAB } 14,2 \% \\
\text { - } \text { CIP/CIM } 36,0 \% \\
\text { - } \text { SHT } 9,5 \% \\
\text { - zerebrale Hypoxie } 11,1 \% \\
\text { - } \text { sonstige neurologische Diagnose } 9,5 \% \\
\text { - } \text { keine neurologische Diagnose } 2,5 \%\end{array}$ \\
\hline Weaningdauer & $21,8 \pm 20,5$ Tage (Range $1-259$ Tage) \\
\hline Weaning-Outcome & $\begin{array}{l}\text { - erfolgreiches Weaning (gemäß dem definierten Weaning- } \\
\text { kriterium: mindestens } 7 \text { Tage ohne invasive bzw. nicht } \\
\text { invasive maschinelle Atemunterstützung) } 69,8 \% \\
\text { - beatmet entlassen } 18,4 \% \text { (davon } 61,3 \% \text { in die rehabilita- } \\
\text { tive oder außerklinische Weiterversorgung und 38,7\% in } \\
\text { eine andere, spezialisierte Krankenhausbehandlung) } \\
\text { - verstorben } 16,6 \% \text { (davon 70,9\% während des Weaning- } \\
\text { prozesses und 29,1\% nach erfolgreichem Weaning) }\end{array}$ \\
\hline
\end{tabular}

$\mathrm{ICB}=$ intrazerebrale Blutung, $\mathrm{SAB}=$ Subarachnoidalblutung, $\mathrm{CIP}=$ Critical-IllnessPolyneuropathie, $\mathrm{CIM}=$ Critical-Illness-Polymyopathie 
Tab. 2: Ergebnisse der Weaningstudien aus dem Gebiet der neurologisch-neurochirurgischen Frührehabilitation im Vergleich

\begin{tabular}{c|l|l|l|l}
\hline Fallzahl & Entwöhnt entlassen (\%) & Beatmet entlassen (\%) & Verstorben (\%) & Autor \\
\hline 193 & 66,8 & 20,2 & 12,9 & Hoffmann et al. 2006 [9] \\
\hline 133 & 78,2 & $5,3 \mathrm{~b} / ? \mathrm{c}$ & $?$ & Bertram und Brandt 2007 [3] \\
\hline 82 & 68,3 & $6,1 \mathrm{~b} / 19,5 \mathrm{c}$ & 6,1 & Rollnik et al. 2010 [25] \\
\hline 1.486 & 64,9 & $11,3 \mathrm{~b} / 7,1 \mathrm{c}$ & 16,6 & Oehmichen et al. 2012 [19] \\
\hline 644 & 59,5 & $9,8 \mathrm{~b}(8,2 \mathrm{~d} / 1,6 \mathrm{a}) / 7,8 \mathrm{c}$ & 23,0 & Oehmichen et al. 2013 [17] \\
\hline 192 & 65,1 & $8,3 \mathrm{~b} / 5,2 \mathrm{c}$ & 21,4 & Pohl et al. 2016 [22] \\
\hline
\end{tabular}

a: Patienten mit noninvasiver Beatmungsform; b: in Weiterversorgung; c: in Krankenhaus; d: invasive Beatmungsform

15, 31]. Während der Spontanatmungsphasen wird der Patient vom Beatmungsgerät diskonnektiert. Durch die zwischengeschaltete passagere kontrollierte Beatmung soll eine Erholung der Atemmuskulatur erreicht werden $[4,12,31]$.

Diese Weaningprotokolle finden also bei allen Patienten in neurologischen Weaningzentren Anwendung, unabhängig ob es sich bei den Patienten um eine zentralnervöse Atemstörung, eine neuromuskuläre Schwäche, eine primär hypoxische Störung infolge pulmonaler Insuffizienz oder um Mischformen handelt [17].

\section{Definition des erfolgreichen Weaning von der Beatmung}

In der pneumologischen Literatur wird das Weaningende uneinheitlich definiert. Obwohl nicht invasive Beatmung als vollwertige Beatmung im DRG-System anerkannt wird [11], wird in der pneumologischen Literatur häufig die Auffassung vertreten, dass eine Beatmungsentwöhnung bereits nach dem Wechsel von invasiver auf (nächtliche) nicht invasive Beatmung erreicht ist $[2,14,30,31]$. Somit wird die erfolgreiche Entwöhnung von der Beatmung mit der Entfernung des invasiven Beatmungszugangs (Endotrachealtubus/Trachealkanüle) gleichgesetzt. Im Gegensatz zu pneumologisch erkrankten Patienten besteht die Indikation für die Trachealkanüle bei neurologisch Erkrankten nicht nur aufgrund der prolongierten invasiven Beatmung, sondern oftmals auch aufgrund der Dysphagie mit Aspirationsgefahr (s.u.). Somit bestehen prinzipiell zwei Indikationen für den Verbleib der Trachealkanüle. Das Weaning von der Beatmungsmaschine ist somit beendet, wenn keine maschinelle Atemunterstützung mehr notwendig ist. Die Entfernung der Trachealkanüle erfolgt, wenn keine Aspirationsgefahr mehr besteht. Hierin besteht einer der Hauptunterschiede zwischen den pneumologischen Patienten, die nur in seltenen Fällen derartige Schluckstörungen aufweisen, und den Patienten, die in der neurologisch-neurochirurgischen Frührehabilitation behandelt werden. Der Einsatz der nicht invasiven Beatmung spielt in der neurologisch-neurochirurgischen Frührehabilitation eine untergeordnete Rolle, da in der überwiegenden Zahl der Fälle Kontraindikationen gegen diese Beatmungsform vorliegen. Kontraindikationen neben den klassischen sind die Tatsachen, dass in der NNFR häufig mangelnde Kooperation, Sekretretention mit mangelndem Hustenstoß, fehlende Schutzreflexe, zentrale Atemantriebsstörungen, komplexe Dysphagie mit Aspirationsrisiko und/oder Störungen der Magenmotilität mit häufigem Erbrechen bestehen.

Zudem hat auch die nicht invasive Beatmung ein spezifisches Behandlungsrisiko und generiert, wie die invasive Beatmung, einen maschinellen und personellen Aufwand. Deshalb werden Patienten der NNFR in aller Regel als entwöhnt betrachtet, wenn die Patienten ohne jede maschinelle Unterstützung bei der Atmung auskommen.

\section{Fazit}

Neurologische Patienten mit prolongiertem Weaning, die nicht in der Primärversorgung von der Beatmung entwöhnt werden konnten, benötigen eine nahtlose Fortführung rehabilitativer Maßnahmen in der Sekundärversorgung unter der Berücksichtigung einer vorhandenen intensivmedizinischen Betreuung in einem spezialisierten Zentrum. Die Beatmungsentwöhnung in den Strukturen der NNFR wird inzwischen deutschlandweit erfolgreich praktiziert. Auch die publizierten Studien belegen die Bedeutung der NNFR als neurologische Weaningzentren.

Das Weaning von der Beatmung von Patienten der NNFR weist im Vergleich zum Weaning nicht neurologischer Patienten einige Besonderheiten auf. Dazu zählen bestimmte Prozesse (z. B. Trachealkanülen- und Dysphagiemanagement) und Konzepte (z.B. der kognitiven oder motorischen Rehabilitation), die grundsätzlich in der NNFR etabliert sind.

Das Weaning in spezialisierten neurologischen Abteilungen oder Kliniken stellt somit einen wesentlichen Baustein im Gesamtbehandlungskonzept von Patienten mit primären und sekundären neurologischneurochirurgischen Erkrankungen dar. Die Vergleichbarkeit der Ergebnisse verschiedener Weaningzentren kann nur hergestellt werden, wenn ein einheitlicher und interdisziplinärer Datensatz entwickelt wird. Dafür seien gleichartige und nachvollziehbare Struktur-, Prozess- und Ergebnisdaten festzulegen. Hierbei besteht 
vielfältiger Klärungsbedarf insbesondere in Bezug auf die Beschreibung der Aufnahmekriterien, der Weaningdefinition und der Ergebnisevaluation in Bezug auf das Therapieziel. Die derzeit in Vorbereitung befindliche Leitlinie zum Weaning in der NNFR der Deutschen Gesellschaft für Neurorehabilitation (DGNR) verspricht hier Aufschluss in Bezug auf einige der beschriebenen Unklarheiten.

\section{Literatur}

1. Bagley PH, Cooney E. A community-based regional ventilator weaning unit: development and outcomes. Chest 1997; 111: 1024-1029.

2. Barchfeld T, Dellweg D, Böckling S et al. Entwöhnung von der Langzeitbeatmung: Daten eines Weaningzentrums von 2007 bis 2011. Dtsch Med Wochenschr 2014; 139: 527-533.

3. Bertram M, Brandt T. Neurologisch-neurochirurgische Frührehabilitation: Eine aktuelle Bestandsaufnahme. Nervenarzt 2007; 78: 1160-1174.

4. Bertram M, Brandt T. Neurologische Frührehabilitation bei beatmeten Patienten mit ZNS-Störungen. Intensivmedizin up2date 2013; 9: 53-71.

5. Boles JM, Bion J, Connors A et al. Weaning from mechanical ventilation. Eur Respir J 2007; 29: 1033-1056.

6. Carson SS, Bach PB, Brzozowski L et al. Outcomes after longterm acute care. An analysis of 133 mechanically ventilated patients. Am J Respir Crit Care Med 1999; 159: 1568-1573.

7. Coplin WM, Pierson DJ, Cooley KD et al. Implications of extubation delay in brain-injured patients meeting standard weaning criteria. Am J Respir Crit Care Med 2000; 161: 1530-1536.

8. Dasgupta A, Rice R, Mascha E et al. Four-year experience with a unit for long-term ventilation (respiratory special care unit) at the Cleveland Clinic Foundation. Chest 1999; 116: 447-455.

9. Hoffmann B, Karbe H, Krusch C et al. Patientencharakteristika in der neurologisch/neurochirurgischen Frührehabilitation (Phase B): Eine multizentrische Erfassung im Jahr 2002 in Deutschland. Akt Neurol 2006; 33: 287-296.

10. Indihar FJ. A 10-year report of patients in a prolonged respiratory care unit. Minn Med 1991; 74: 23-27.

11. Institut für das Entgeltsystem im Krankenhaus (InEK $\mathrm{GmbH})$. Deutsche Kodierrichtlinien - Allgemein und spezielle Kodierrichtlinien für die Verschlüsselung von Krankheiten und Prozeduren. Köln: Deutsche Ärzte-Verlag; 2015.

12. Krishnan JA, Moore DM, Robeson C et al. A prospective, controlled trial of a protocol-based strategy to discontinue mechanical ventilation. Am J Respir Crit Care Med 2004; 169: 673-678.

13. Latriano B, McCauley P, Astiz ME et al. Non-ICU care of hemodynamically stable mechanically ventilated patients. Chest 1996; 109: 1591-1596.

14. MacIntyre NR, Epstein SK, Carson S et al. Management of patients requiring prolonged mechanical ventilation: report of a NAMDRC consensus conference. Chest 2005; 128: 3937-3954.

15. MacIntyre NR, Cook DJ, Ely EW, Jr. et al. Evidence-based guidelines for weaning and discontinuing ventilatory support: a collective task force facilitated by the American College of Chest Physicians; the American Association for Respiratory Care; and the American College of Critical Care Medicine. Chest 2001; 120: 375S-395S.

16. Oehmichen F, Ragaller M. Beatmungsentwöhnung bei Chronisch-Kritisch-Kranken. Intensiv- und Notfallbehandlung 2012; 37: 118-126.

17. Oehmichen F, Zäumer K, Ragaller M et al. Anwendung eines standardisierten Spontanatmungsprotokolls - Erfahrungen in einem Weaning-Zentrum mit neurologischem Schwerpunkt. Nervenarzt 2013; 84: 962-972.
18. Oehmichen F, Pohl M, Schlosser R et al. Critical-IllnessPolyneuropathie und -Polymyopathie: Wie sicher ist die klinische Diagnose bei Patienten mit Weaning-Versagen? Nervenarzt 2012; 83: 220-225.

19. Oehmichen F, Ketter G, Mertl-Rotzer M et al. Beatmungsentwöhnung in neurologischen Weaningzentren - Eine Bestandsaufnahme der Arbeitsgemeinschaft Neurologischneurochirurgische Frührehabilitation. Nervenarzt 2012; 83: 1300-1307.

20. Pohl M, Mehrholz J. Auf einer Intensivstation erworbenes Schwächesyndrom - Langzeitkomplikationen. Neurorehabil Neural Repair 2013; 1: 17-20.

21. Pohl M, Wallsch CW, Platz T et al. Rehabilitationsverlauf von Patienten der Phase B/Frühreha: Ergebnisse einer neuen Multizenterstudie aus 2014. In: 5. Gemeinsame Jahrestagung der Deutschen Gesellschaft für Neurorehabilitation e. V. und der Deutschen Gesellschaft für Neurotraumatologie und Klinische Neurorehabilitation e. V. Singen; 2014

22. Pohl M, Bertram M, Bucka C et al. Rehabilitationsverlauf von Patienten in der neurologisch-neurochirurgischen Frührehabilitation: Ergebnisse einer multizentrischen Erfassung im Jahr 2014 in Deutschland. Nervenarzt 2016; Epub ahead 18.04.2016.

23. Ponfick M, Wiederer R, Bosl $\mathrm{K}$ et al. The influence of weaning duration on rehabilitative outcome in early neurological rehabilitation. NeuroRehabilitation 2014; 34: 493-498.

24. Ponfick M, Bösl K, Lüdemann-Podubecka J et al. Erworbe ne Muskelschwäche des kritisch Kranken: Pathogenese, Behandlung, Rehabilitation, Outcome. Nervenarzt 2014; 85: 195-204

25. Rollnik JD, Berlinghof $\mathrm{K}$, Lenz $\mathrm{O}$ et al. Beatmung in der neurologischen Frührehabilitation. Akt Neurol 2010; 37: 316-318.

26. Scheinhorn DJ, Chao DC, Stearn-Hassenpflug M et al. PostICU mechanical ventilation: treatment of 1,123 patients at a regional weaning center. Chest 1997; 111: 1654-1659.

27. Scheinhorn DJ, Hassenpflug MS, Votto JJ et al. Post-ICU mechanical ventilation at 23 long-term care hospitals: a multicenter outcomes study. Chest 2007; 131: 85-93.

28. Schönhofer B, Haidl P, Kemper P et al. Entwöhnung vom Respirator (»Weaning») bei Langzeitbeatmung - Ergebnisse bei Patienten in einem Entwöhnungszentrum. Dtsch Med Wochenschr 1999; 124: 1022-1028.

29. Schönhofer B, Euteneuer S, Nava S et al. Survival of mechanically ventilated patients admitted to a specialised weaning centre. Intensive Care Med 2002; 28: 908-916.

30. Schönhofer B, Berndt C, Achtzehn U et al. Entwöhnung von der Beatmungstherapie - Eine Erhebung zur Situation pneumologischer Beatmungszentren in Deutschland. Dtsch Med Wochenschr 2008; 133: 700-704.

31. Schönhofer B, Geiseler J, Dellweg D et al. Prolongiertes Weaning - S2k-Leitlinie herausgegeben von der Deutschen Gesellschaft für Pneumologie und Beatmungsmedizin e.V. Pneumologie 2014; 68: 19-75.

32. Wallesch CW. Die Rehabilitation (noch) nicht rehabilitationsfähiger Patienten in der neurologischen Frührehabilitation. Fortschr Neurol Psychiatr 2015; 83: 257-258.

33. Wallesch CW. Die neurologische Frührehabilitation Phase B als neuer Subsektor der akutstationären Behandlung. Akt Neurol 2016; 43: 280-284.

\section{Interessenvermerk}

Der Autor gibt an, dass kein Interessenkonflikt vorliegt.

\section{Korrespondenzadresse:}

Prof. Dr. Marcus Pohl

HELIOS Klinik Schloss Pulsnitz GmbH

Wittgensteiner Str. 1

01896 Pulsnitz

Marcus.Pohl@helios-kliniken.de 